\title{
MS22-05 | Using Crystal Structure, an Improved Electrochemical Method and Computational DFt Studies to Understand the Medicinal Properties of
}

\section{Celastraceae Species of Plants}

Caruso, Francesco (Vassar College, Poughkeepsie, USA); Belli, Stuart (Vassar College, Poughkeepsie, USA); Singh, Manrose (Vassar College, Poughkeepsie, USA); Berinato, Molly (Vassar College, Poughkeepsie, USA); Chavez, Haydee (Universidad Nacional San Luis Gonzaga, Ica, PER); Rossi, Miriam (Vassar College, Poughkeepsie, USA)

Members of the Celastraceae family all over the world have remarkable medicinal properties. We describe the results of diverse experimental and computational methodologies on two members of this family to understand their pharmacological activity. First, we focus on celastrol, a triterpenoid extracted from Tripterygium wilfordii Hook F., a perennial vine of Celastraceae family (Chinese name "lei gong teng"). This plant (and compound) is often used in traditional medicine as a remedy for inflammatory and autoimmune diseases. We describe features from the crystal structure of the molecule, and together with results of computational docking and DFT studies, attempt to elucidate celastrol's biological activities on a molecular level. Additionally, our research focuses on determining the antioxidant effectiveness of pure celastrol using a recently improved voltammetry method with a rotating ringdisk electrode (RRDE). This method tests the reactivity of celastrol towards the superoxide radical anion. This is particularly interesting as the superoxide radical is produced by the body under oxidative stress and is considered a damaging species. Next, the results of similar electrochemistry experiments to detect the antioxidant reactivity of an ethanolic extract of leaves from a related member of the medically rich Celastraceae family, the Maytenus octogona tree, with superoxide radical are described. 